\title{
Approximate Solution of Inverse Problem for Elliptic Equation with Overdetermination
}

\author{
Charyyar Ashyralyyev ${ }^{1,2}$ and Mutlu Dedeturk ${ }^{1}$ \\ ${ }^{1}$ Department of Mathematical Engineering, Gumushane University, 29100 Gumushane, Turkey \\ ${ }^{2}$ TAU, Gerogly Street 143, 74400 Ashgabat, Turkmenistan \\ Correspondence should be addressed to Mutlu Dedeturk; mutludedeturk@gumushane.edu.tr
}

Received 5 July 2013; Revised 14 August 2013; Accepted 20 August 2013

Academic Editor: Abdullah Said Erdogan

Copyright ( 2013 C. Ashyralyyev and M. Dedeturk. This is an open access article distributed under the Creative Commons Attribution License, which permits unrestricted use, distribution, and reproduction in any medium, provided the original work is properly cited.

\begin{abstract}
A finite difference method for the approximate solution of the inverse problem for the multidimensional elliptic equation with overdetermination is applied. Stability and coercive stability estimates of the first and second orders of accuracy difference schemes
\end{abstract} for this problem are established. The algorithm for approximate solution is tested in a two-dimensional inverse problem.

\section{Introduction}

It is well known that inverse problems arise in various branches of science (see $[1,2])$. The theory and applications of well-posedness of inverse problems for partial differential equations have been studied extensively by many researchers (see, e.g., [3-17] and the references therein). One of the effective approaches for solving inverse problem is reduction to nonlocal boundary value problem (see, e.g., $[6,8,11]$ ). Well-posedness of the nonlocal boundary value problems of elliptic type equations was investigated in [18-25] (see also the references therein).

In [4], Orlovsky proved existence and uniqueness theorems for the inverse problem of finding a function $u$ and an element $p$ for the elliptic equation in an arbitrary Hilbert space $H$ with the self-adjoint positive definite operator $A$ :

$$
\begin{gathered}
-u_{t t}(t)+A u(t)=f(t)+p, \quad 0<t<T, \\
u(0)=\varphi, \quad u(T)=\psi, \quad u(\lambda)=\xi, \quad 0<\lambda<T .
\end{gathered}
$$

In [11], the authors established stability estimates for this problem and studied inverse problem for multidimensional elliptic equation with overdetermination in which the Dirichlet condition is required on the boundary.

In present work, we study inverse problem for multidimensional elliptic equation with Dirichlet-Neumann boundary conditions.
Let $\Omega=(0, \ell) \times \cdots \times(0, \ell)$ be the open cube in the $n$ dimensional Euclidean space with boundary $S$ and $\bar{\Omega}=\Omega \cup$ $S$. In $[0, T] \times \Omega$, we consider the inverse problem of finding functions $u(t, x)$ and $p(x)$ for the multidimensional elliptic equation

$$
\begin{array}{cc}
-u_{t t}(t, x)-\sum_{r=1}^{n}\left(a_{r}(x) u_{x_{r}}\right)_{x_{r}}+\delta u(t, x)=f(t, x)+p(x), \\
x=\left(x_{1}, \ldots, x_{n}\right) \in \Omega, \quad 0<t<T, & \\
u(0, x)=\varphi(x), \quad u(T, x)=\psi(x), \quad u(\lambda, x)=\xi(x), \\
x \in \bar{\Omega}, \\
\frac{\partial u(t, x)}{\partial \vec{n}}=0, \quad x \in S, 0 \leq t \leq T .
\end{array}
$$

Here, $0<\lambda<T$ and $\delta>0$ are known numbers, $a_{r}(x)(x \in \Omega), \varphi(x), \psi(x), \xi(x)(x \in \bar{\Omega})$, and $f(t, x)(t \in$ $(0, T), x \in \Omega)$ are given smooth functions, and also $a_{r}(x) \geq$ $a>0(x \in \Omega)$.

The aim of this paper is to investigate inverse problem (2) for multidimensional elliptic equation with DirichletNeumann boundary conditions. We obtain well-posedness of problem (2). For the approximate solution of problem (2), we construct first and second order of accuracy in 
$t$ and difference schemes with second order of accuracy in space variables. Stability and coercive stability estimates for these difference schemes are established by applying operator approach. The modified Gauss elimination method is applied for solving these difference schemes in a two-dimensional case.

The remainder of this paper is organized as follows. In Section 2, we obtain stability and coercive stability estimates for problem (2). In Section 3, we construct the difference schemes for (2) and establish their well-posedness. In Section 4 , the numerical results in a two-dimensional case are presented. Section 5 is conclusion.

\section{Well-Posedness of Inverse Problem with Overdetermination}

It is known that the differential expression [26]

$$
A^{x} u(x)=-\sum_{r=1}^{n}\left(a_{r}(x) u_{x_{r}}\right)_{x_{r}}+\delta u(x)
$$

defines a self-adjoint positive definite operator $A^{x}$ acting on $L_{2}(\bar{\Omega})$ with the domain $D\left(A^{x}\right)=\left\{u(x) \in W_{2}^{2}(\bar{\Omega}), \partial u / \partial \vec{n}=\right.$ 0 on $S$ \}.

Let $H$ be the Hilbert space $L_{2}(\bar{\Omega})$. By using abstract Theorems 2.1 and 2.2 of paper [11], we get the following theorems about well-posedness of problem (2).

Theorem 1. Assume that $A^{x}$ is defined by formula (3), $\varphi, \xi, \psi \in$ $D\left(A^{x}\right)$. Then, for the solutions $(u, p)$ of inverse boundary value problem (2), the stability estimates are satisfied:

$$
\begin{aligned}
& \|u\|_{C\left(L_{2}(\bar{\Omega})\right)} \\
& \leq\left[M\|\varphi\|_{L_{2}(\bar{\Omega})}+\|\psi\|_{L_{2}(\bar{\Omega})}+\|\xi\|_{L_{2}(\bar{\Omega})}+\|f\|_{C\left(L_{2}(\bar{\Omega})\right)}\right], \\
& \left\|\left(A^{x}\right)^{-1} p\right\|_{L_{2}(\bar{\Omega})} \leq M\left[\|\varphi\|_{L_{2}(\bar{\Omega})}\right. \\
& \left.+\|\xi\|_{L_{2}(\bar{\Omega})}+\|f\|_{C\left(L_{2}(\bar{\Omega})\right)}\right], \\
& \|p\|_{L_{2}(\bar{\Omega})} \leq M\left[\left\|A^{x} \varphi\right\|_{L_{2}(\bar{\Omega})}+\left\|A^{x} \psi\right\|_{L_{2}(\bar{\Omega})}\right. \\
& \left.+\left\|A^{x} \xi\right\|_{L_{2}(\bar{\Omega})}+\frac{1}{\alpha(1-\alpha)}\|f\|_{\mathscr{C}_{0 T}^{\alpha, \alpha}\left(L_{2}(\bar{\Omega})\right)}\right],
\end{aligned}
$$

where $M$ is independent of $\alpha, \varphi(x), \xi(x), \psi(x)$, and $f(t, x)$.

Here, $\mathscr{C}_{0 T}^{\alpha, \alpha}\left(L_{2}(\bar{\Omega})\right)$ is the space obtained by completion of the space of all smooth $L_{2}(\bar{\Omega})$-valued functions $\rho$ on $[0, T]$ with the norm

$$
\begin{aligned}
& \|\rho\|_{\mathscr{C}_{0 T}^{\alpha, \alpha}\left(L_{2}(\bar{\Omega})\right)} \\
& =\|\rho\|_{\mathscr{C}\left(L_{2}(\bar{\Omega})\right)} \\
& \quad+\sup _{0 \leq t<t+\tau \leq T} \frac{(t+\tau)^{\alpha}(T-t)^{\alpha}\|\rho(t+\tau)-\rho(t)\|_{L_{2}(\bar{\Omega})}}{\tau^{\alpha}} .
\end{aligned}
$$

Theorem 2. Assume that $A^{x}$ is defined by formula (3), $\varphi, \psi, \xi \in D\left(A^{x}\right)$. Then, for the solution of inverse boundary value problem (2), coercive stability estimate

$$
\begin{gathered}
\left\|u^{\prime \prime}\right\|_{\mathscr{C}_{0 T}^{\alpha, \alpha}\left(L_{2}(\bar{\Omega})\right)}+\|u\|_{\mathscr{C}_{0 T}^{\alpha, \alpha}\left(W_{2}^{2}(\bar{\Omega})\right)}+\|p\|_{L_{2}(\bar{\Omega})} \\
\leq M\left[\frac{1}{\alpha(1-\alpha)}\|f\|_{\mathscr{C}_{0 T}^{\alpha, \alpha}\left(L_{2}(\bar{\Omega})\right)}+\|\varphi\|_{W_{2}^{2}(\bar{\Omega})}\right. \\
\left.+\|\psi\|_{W_{2}^{2}(\bar{\Omega})}+\|\xi\|_{W_{2}^{2}(\bar{\Omega})}\right]
\end{gathered}
$$

holds, where $M$ is independent of $\alpha, \varphi(x), \xi(x), \psi(x)$, and $f(t, x)$.

\section{Difference Schemes and Their Well-Posedness}

Suppose that $A^{x}$ is defined by formula (3). Then (see [26]), $C=(1 / 2)\left(\tau A^{x}+\sqrt{4 A^{x}+\tau^{2}\left(A^{x}\right)^{2}}\right)$ is a self-adjoint positive definite operator and $R=(I+\tau C)^{-1}$ which is defined on the whole space $H=L_{2}(\bar{\Omega})$ is a bounded operator. Here, $I$ is the identity operator.

Now we present the following lemmas, which will be used later.

Lemma 3. The following estimates are satisfied (see [27]):

$$
\begin{gathered}
\left\|R^{k}\right\|_{H \rightarrow H} \leq M\left(1+\delta^{1 / 2} \tau\right)^{-k}, \quad \delta>0, \\
\left\|C R^{k}\right\|_{H \rightarrow H} \leq \frac{M}{k \tau}, \quad k \geq 1, \\
\left\|\left(I-R^{2 N}\right)^{-1}\right\|_{H \rightarrow H} \leq M .
\end{gathered}
$$

Lemma 4. For $1 \leq l \leq N-1$ and for the operator $S=R^{2 N}+$ $R^{l}-R^{2 N-l}+R^{N-l}-R^{N+l}$, the operator I $S$ has an inverse $G=(I-S)^{-1}$ and the estimate

$$
\|G\|_{H \rightarrow H} \leq M
$$

is satisfied, where $M$ does not depend on $\tau$.

Proof of Lemma 4 is based on Lemma 3 and representation

$$
\begin{aligned}
Q & =I-R^{2 N}-R^{l}+R^{2 N-l}-R^{N-l}+R^{N+l} \\
& =\left(I-R^{N-l}\right)\left(I-R^{N}\right)\left(I-R^{l}\right) .
\end{aligned}
$$

Lemma 5. For $1 \leq l \leq N-1$ and for the operator

$$
\begin{aligned}
S_{1}= & R^{2 N}-\left(\frac{\lambda}{\tau}-l-1\right)\left(R^{l}-R^{2 N-l}+R^{N-l}-R^{N+l}\right) \\
& +\left(\frac{\lambda}{\tau}-l\right)\left(R^{l+1}-R^{2 N-l-1}+R^{N-l-1}-R^{N+l+1}\right)
\end{aligned}
$$


the operator $I-S_{1}$ has an inverse

$$
\begin{aligned}
G_{1}=( & I-R^{2 N}+\left(\frac{\lambda}{\tau}-l-1\right) \\
& \times\left(R^{l}-R^{2 N-l}+R^{N-l}-R^{N+l}\right) \\
& \left.-\left(\frac{\lambda}{\tau}-l\right)\left(R^{l+1}-R^{2 N-l-1}+R^{N-l-1}-R^{N+l+1}\right)\right)^{-1}
\end{aligned}
$$

and the estimate

$$
\left\|G_{1}\right\|_{H \rightarrow H} \leq M
$$

is valid, where $M$ is independent of $\tau$.

Proof. We have that

$$
G_{1}-G=G_{1} G K
$$

where

$$
\begin{aligned}
K= & -\left(\frac{\lambda}{\tau}-l\right)\left(R^{l}-R^{2 N-l}+R^{N-l}-R^{N+l}\right) \\
& +\left(\frac{\lambda}{\tau}-l\right)\left(R^{l+1}-R^{2 N-l-1}+R^{N-l-1}-R^{N+l+1}\right) .
\end{aligned}
$$

By using estimates of Lemma 3, we have that

$$
\begin{array}{r}
\|K\|_{H \rightarrow H}=\|-\left(\frac{\lambda}{\tau}-l\right)\left(R^{l}-R^{2 N-l}+R^{N-l}-R^{N+l}\right) \\
+\left(\frac{\lambda}{\tau}-l\right)\left(R^{l+1}-R^{2 N-l-1}+R^{N-l-1}\right. \\
\left.-R^{N+l+1}\right) \|_{H \rightarrow H} \leq M_{1} \tau,
\end{array}
$$

where $M_{1}$ is independent of $\tau$. Using the triangle inequality, formula (13), and estimates (8) and (15), we obtain

$$
\begin{aligned}
\left\|G_{1}\right\|_{H \rightarrow H} & =\|G\|_{H \rightarrow H}+\left\|G_{1}\right\|_{H \rightarrow H}\|G\|_{H \rightarrow H} \\
& \leq M+\left\|G_{1}\right\|_{H \rightarrow H} M M_{1} \tau
\end{aligned}
$$

for sufficiently small positive $\tau$. From that it follows estimate (11). Lemma 5 is proved.

Further, we discretize problem (2) in two steps. In the first step, we define the grid spaces

$$
\begin{gathered}
\widetilde{\Omega}_{h}=\left\{x=x_{m}=\left(h_{1} m_{1}, \ldots, h_{n} m_{n}\right) ; m=\left(m_{1}, \ldots, m_{n}\right),\right. \\
\left.m_{r}=0, \ldots, M_{r}, h_{r} M_{r}=\ell, r=1, \ldots, n\right\}, \\
\Omega_{h}=\widetilde{\Omega}_{h} \cap \Omega, \quad S_{h}=\widetilde{\Omega}_{h} \cap S .
\end{gathered}
$$

Introduce the Hilbert space $L_{2 h}=L_{2}\left(\widetilde{\Omega}_{h}\right)$ and $W_{2 h}^{2}=$ $W_{2}^{2}\left(\widetilde{\Omega}_{h}\right)$ of grid functions $\rho^{h}(x)=\left\{\rho\left(h_{1} m_{1}, \ldots, h_{n} m_{n}\right)\right\}$, defined on $\widetilde{\Omega}_{h}$, equipped with the norms

$$
\begin{gathered}
\left\|\rho^{h}\right\|_{L_{2 h}}=\left(\sum_{x \in \widetilde{\Omega}_{h}}\left|\rho^{h}(x)\right|^{2} h_{1}, \ldots, h_{n}\right)^{1 / 2}, \\
\left\|\rho^{h}\right\|_{W_{2 h}^{2}}=\left\|\rho^{h}\right\|_{L_{2 h}}+\left(\sum_{x \in \widetilde{\Omega}_{h}} \sum_{r=1}^{n}\left|\left(\rho^{h}\right)_{x_{r}}\right|^{2} h_{1}, \ldots, h_{n}\right)^{1 / 2} \\
+\left(\sum_{x \in \widetilde{\Omega}_{h}} \sum_{r=1}^{n}\left|\left(\rho^{h}(x)\right)_{x_{r} \bar{x}_{r}, m_{r}}\right|^{2} h_{1}, \ldots, h_{n}\right)^{1 / 2},
\end{gathered}
$$

respectively.

To the differential operator $A^{x}$ generated by problem (2) we assign the difference operator $A_{h}^{x}$ defined by formula (3), acting in the space of grid functions $u^{h}(x)$, satisfying the condition $D^{h} u^{h}(x)=0$ for all $x \in S_{h}$. Here, $D^{h} u^{h}(x)$ is an approximation of $\partial u / \partial \vec{n}$.

By using $A_{h}^{x}$, for obtaining $u^{h}(t, x)$ functions, we arrive at problem

$$
\begin{array}{r}
-\frac{d^{2} u^{h}(t, x)}{d t^{2}}+A_{h}^{x} u^{h}(t, x)=f^{h}(t, x)+p^{h}(x), \\
0<t<T, x \in \Omega_{h},
\end{array}
$$

$u^{h}(0, x)=\varphi^{h}(x), \quad u^{h}(\lambda, x)=\xi^{h}(x), \quad u^{h}(T, x)=\psi^{h}(x)$,

$$
x \in \widetilde{\Omega}_{h} .
$$

For finding a solution $u^{h}(t, x)$ of problem (19) we apply the substitution

$$
u^{h}(t, x)=v^{h}(t, x)+\left(A_{h}^{x}\right)^{-1} p^{h}(x),
$$

where $v^{h}(t, x)$ is the solution of nonlocal boundary value problem; a system of ordinary differential equations

$$
\begin{gathered}
-\frac{d^{2} v^{h}(t, x)}{d t^{2}}+A_{h}^{x} v^{h}(t, x)=f^{h}(t, x), \\
0<t<T, \quad x \in \Omega_{h}, \\
v^{h}(0, x)-v^{h}(\lambda, x)=\varphi^{h}(x)-\xi^{h}(x), \quad x \in \widetilde{\Omega}_{h}, \\
v^{h}(T, x)-v^{h}(\lambda, x)=\psi^{h}(x)-\xi^{h}(x), \quad x \in \widetilde{\Omega}_{h}
\end{gathered}
$$

and unknown function $p^{h}(x)$ is defined by formula

$$
p^{h}(x)=A_{h}^{x} \varphi^{h}(x)-A_{h}^{x} v^{h}(0, x), \quad x \in \widetilde{\Omega}_{h} .
$$

Thus, we consider the algorithm for solving problem (19) which includes three stages. In the first stage, we get 
the nonlocal boundary value problem (21) and obtain $v^{h}(t, x)$. In the second stage, we put $t=0$ and find $v^{h}(0, x)$. Then, using (22), we obtain $p^{h}(x)$. Finally, in the third stage, we use formula (20) for obtaining the solution $u^{h}(t, x)$ of problem (19).

In the second step, we approximate (19) in variable $t$. Let $[0, T]_{\tau}=\left\{t_{k}=k \tau, k=1, \ldots, N, N \tau=T\right\}$ be the uniform grid space with step size $\tau>0$, where $N$ is a fixed positive integer. Applying the approximate formulas

$$
\begin{aligned}
u^{h}(\lambda, x) & =u^{h}\left(\left[\frac{\lambda}{\tau}\right] \tau, x\right)+o(\tau), \quad x \in \Omega_{h}, \\
u^{h}(\lambda, x)= & u^{h}\left(\left[\frac{\lambda}{\tau}\right] \tau, x\right)+\left(\frac{\lambda}{\tau}-\left[\frac{\lambda}{\tau}\right]\right) \\
& \times\left(u^{h}\left(\left[\frac{\lambda}{\tau}\right] \tau+\tau, x\right)-u^{h}\left(\left[\frac{\lambda}{\tau}\right] \tau, x\right)\right) \\
& +o\left(\tau^{2}\right), \quad x \in \Omega_{h}
\end{aligned}
$$

for $u^{h}(\lambda, x)=\xi^{h}(x)$, problem (19) is replaced by first order of accuracy difference scheme

$$
\begin{gathered}
-\frac{u_{k+1}^{h}(x)-2 u_{k}^{h}(x)+u_{k-1}^{h}(x)}{\tau^{2}}+A_{h}^{x} u_{k}^{h}(x) \\
=f_{k}^{h}(x)+p^{h}(x), \\
f_{k}^{h}(x)=f^{h}\left(t_{k}, x\right), \quad t_{k}=k \tau, \\
1 \leq k \leq N-1, \quad x \in \Omega_{h}, \\
u_{0}^{h}(x)=\varphi^{h}(x), \quad x \in \widetilde{\Omega}_{h}, \\
u_{l}^{h}(x)=\xi^{h}(x), \quad x \in \widetilde{\Omega}_{h}, \\
u_{N}^{h}(x)=\psi^{h}(x), \quad x \in \widetilde{\Omega}_{h}, \quad l=\left[\frac{\lambda}{\tau}\right]
\end{gathered}
$$

and second order of accuracy difference scheme

$$
\begin{gathered}
-\frac{u_{k+1}^{h}(x)-2 u_{k}^{h}(x)+u_{k-1}^{h}(x)}{\tau^{2}}+A_{h}^{x} u_{k}^{h}(x) \\
=f_{k}^{h}(x)+p^{h}(x), \\
f_{k}^{h}(x)=f^{h}\left(t_{k}, x\right), \quad t_{k}=k \tau, \\
1 \leq k \leq N-1, \quad x \in \Omega_{h}, \\
u_{0}^{h}(x)=\varphi^{h}(x), \quad x \in \widetilde{\Omega}_{h}, \\
u_{l}^{h}(x)+\left(\frac{\lambda}{\tau}-l\right)\left(u_{l+1}^{h}(x)-u_{l}^{h}(x)\right)=\xi^{h}(x), \\
u_{N}^{h}(x)=\psi^{h}(x), \quad x \in \widetilde{\Omega}_{h}, \quad l=\left[\frac{\lambda}{\tau}\right] .
\end{gathered}
$$

For approximate solution of nonlocal problem (21), we have first order of accuracy difference scheme

$$
\begin{gathered}
-\frac{v_{k+1}^{h}(x)-2 v_{k}^{h}(x)+v_{k-1}^{h}(x)}{\tau^{2}}+A_{h}^{x} v_{k}^{h}(x)=f_{k}^{h}(x), \\
f_{k}^{h}(x)=f^{h}\left(t_{k}, x\right), \quad t_{k}=k \tau, \\
1 \leq k \leq N-1, \quad x \in \Omega_{h}, \\
v_{0}^{h}(x)-v_{l}^{h}(x)=\varphi^{h}(x)-\xi^{h}(x), \quad x \in \widetilde{\Omega}_{h}, \\
v_{N}^{h}(x)-v_{l}^{h}(x)=\psi^{h}(x)-\xi^{h}(x), \quad x \in \widetilde{\Omega}_{h}
\end{gathered}
$$

and second order of accuracy difference scheme

$$
\begin{gathered}
-\frac{v_{k+1}^{h}(x)-2 v_{k}^{h}(x)+v_{k-1}^{h}(x)}{\tau^{2}}+A_{h}^{x} v_{k}^{h}(x)=f_{k}^{h}(x), \\
f_{k}^{h}(x)=f^{h}\left(t_{k}, x\right), \\
t_{k}=k \tau, \quad 1 \leq k \leq N, \quad x \in \Omega_{h}, \\
v_{0}^{h}(x)-\left(\frac{\lambda}{\tau}-l\right) v_{l+1}^{h}(x)+\left(\frac{\lambda}{\tau}-l-1\right) v_{l}^{h}(x) \\
=\varphi^{h}(x)-\xi^{h}(x), \\
v_{N}^{h}(x)-\left(\frac{\lambda}{\tau}-l\right) v_{l+1}^{h}(x)+\left(\frac{\lambda}{\tau}-l-1\right) v_{l}^{h}(x) \\
=\psi^{h}(x)-\xi^{h}(x), \quad x \in \widetilde{\Omega}_{h},
\end{gathered}
$$

respectively.

Theorem 6. Let $\tau$ and $|h|=\sqrt{h_{1}^{2}+\cdots+h_{n}^{2}}$ be sufficiently small positive numbers. Then, for the solutions $\left(\left\{u_{k}^{h}\right\}_{K-1}^{N-1}, p^{h}\right)$ of difference schemes (24) and (25) the stability estimates

$$
\begin{gathered}
\left\|\left\{u_{k}^{h}\right\}_{1}^{N-1}\right\|_{\mathscr{C}_{\tau}\left(L_{2 h}\right)} \leq M\left[\left\|\varphi^{h}\right\|_{L_{2 h}}+\left\|\psi^{h}\right\|_{L_{2 h}}+\left\|\xi^{h}\right\|_{L_{2 h}}\right. \\
\left.+\left\|\left\{f_{k}^{h}\right\}_{1}^{N-1}\right\|_{\mathscr{C}_{\tau}\left(L_{2 h}\right)}\right] \\
\left\|p^{h}\right\|_{L_{2 h}} \leq M\left[\left\|\varphi^{h}\right\|_{W_{2 h}^{2}}+\left\|\psi^{h}\right\|_{W_{2 h}^{2}}+\left\|\xi^{h}\right\|_{W_{2 h}^{2}}\right. \\
\left.+\frac{1}{\alpha(1-\alpha)}\left\|\left\{f_{k}^{h}\right\}_{1}^{N-1}\right\|_{\mathscr{C}_{\tau}\left(L_{2 h}\right)}\right]
\end{gathered}
$$

hold, where $M$ is independent of $\tau, \alpha, h, \varphi^{h}(x), \psi^{h}(x), \xi^{h}(x)$, and $\left\{f_{k}^{h}(x)\right\}_{1}^{N-1}$. 
Theorem 7. Let $\tau$ and $|h|=\sqrt{h_{1}^{2}+\cdots+h_{n}^{2}}$ be sufficiently small positive numbers. Then, for the solutions of difference schemes (24) and (25) the following almost coercive stability estimate

$$
\begin{gathered}
\left\|\left\{\frac{\left.u_{k+1}^{h}-2 u_{k}^{h}+u_{k-1}^{k}\right)}{\tau^{2}}\right\}_{1}^{N-1}\right\|_{\mathscr{C}_{\tau}\left(L_{2 h}\right)} \\
+\left\|\left\{u_{k}^{h}\right\}_{1}^{N-1}\right\|_{\mathscr{C}_{\tau}\left(W_{2 h}^{2}\right)}+\left\|p^{h}\right\|_{L_{2 h}} \\
\leq M\left[\left\|\varphi^{h}\right\|_{W_{2 h}^{2}}+\left\|\psi^{h}\right\|_{W_{2 h}^{2}}+\left\|\xi^{h}\right\|_{W_{2 h}^{2}}\right. \\
\left.+\ln \left(\frac{1}{\tau+h}\right)\left\|\left\{f_{k}^{h}\right\}_{1}^{N-1}\right\|_{\mathscr{C}_{\tau}\left(L_{2 h}\right)}\right]
\end{gathered}
$$

holds, where $M$ is independent of $\tau, \alpha, h, \varphi^{h}(x), \psi^{h}(x), \xi^{h}(x)$, and $\left\{f_{k}^{h}(x)\right\}_{1}^{N-1}$.

Proofs of Theorems 6 and 7 are based on the symmetry property of operator $A^{x}$, on Lemmas 3-5, the formulas

$$
\begin{aligned}
& u_{k}^{h}(x)=\left(I-R^{2 N}\right)^{-1} \\
& \times {\left[\left(\left(R^{k}-R^{2 N-k}\right) v_{0}^{h}(x)\right.\right.} \\
&\left.+\left(R^{N-k}-R^{N+k}\right) v_{N}^{h}(x)\right) \\
&-\left(R^{N-k}-R^{N+k}\right)(I+\tau C)(2 I+\tau C)^{-1} \\
&\left.\times C^{-1} \sum_{i=1}^{N-1}\left(R^{N-i}-R^{N+i}\right) f_{i}^{h}(x) \tau\right] \\
&+(I+\tau C)(2 I+\tau C)^{-1} C^{-1} \\
& \times \sum_{i=1}^{N-1}\left(R^{|k-i|}-R^{k+i}\right) \\
& \times f_{i}^{h}(x) \tau+\varphi^{h}(x)-v_{0}^{h}(x), \\
& p^{h}(x)=A_{h}^{x} \varphi^{h}(x)-A_{h}^{x} v_{0}^{h}(x), \\
& v_{N}^{h}(x)=v_{0}^{h}(x)+\psi^{h}(x)-\varphi^{h}(x),
\end{aligned}
$$

$$
\begin{aligned}
v_{0}^{h}(x)= & -G\left(R^{N-l}-R^{N+l}\right) \\
& \times(I+\tau C)(2 I+\tau C)^{-1} C^{-1} \\
& \times \sum_{i=1}^{N-1}\left(R^{N-i}-R^{N+i}\right) f_{i}^{h}(x) \tau \\
& +G\left(I-R^{2 N}\right)(I+\tau C) \\
& \times(2 I+\tau C)^{-1} C^{-1} \sum_{i=1}^{N-1}\left(R^{|l-i|}-R^{l+i}\right) f_{i}^{h}(x) \tau \\
& +G\left(I-R^{2 N}\right)\left(\varphi^{h}(x)-\xi^{h}(x)\right) \\
& +G\left(R^{N-l}-R^{N+l}\right)\left(\psi^{h}(x)-\varphi^{h}(x)\right),
\end{aligned}
$$

for difference scheme (24),

$$
\begin{aligned}
& v_{0}^{h}(x)=\left(\frac{\lambda}{\tau}-l-1\right) G_{1}\left(R^{N-l}-R^{N+l}\right) \\
& \times(I+\tau C)(2 I+\tau C)^{-1} C^{-1} \\
& \times \sum_{i=1}^{N-1}\left(R^{N-i}-R^{N+i}\right) f_{i}^{h}(x) \tau \\
& -\left(\frac{\lambda}{\tau}-l-1\right) G_{1}\left(I-R^{2 N}\right) \\
& \times(I+\tau C)(2 I+\tau C)^{-1} C^{-1} \\
& \times \sum_{i=1}^{N-1}\left(R^{|l-i|}-R^{l+i}\right) f_{i}^{h}(x) \tau \\
& -\left(\frac{\lambda}{\tau}-l\right) G_{1}\left(R^{N-l-1}-R^{N+l+1}\right) \\
& \times(I+\tau C)(2 I+\tau C)^{-1} C^{-1} \\
& \times \sum_{i=1}^{N-1}\left(R^{N-i}-R^{N+i}\right) f_{i}^{h}(x) \tau \\
& +\left(\frac{\lambda}{\tau}-l\right) G_{1}\left(I-R^{2 N}\right) \\
& \times(I+\tau C)(2 I+\tau C)^{-1} C^{-1} \\
& \times \sum_{i=1}^{N-1}\left(R^{|l+1-i|}-R^{l+1+i}\right) f_{i}^{h}(x) \tau \\
& +G_{1}\left(I-R^{2 N}\right)\left(\varphi^{h}(x)-\xi^{h}(x)\right)
\end{aligned}
$$




$$
\begin{aligned}
& +\left(\left(\frac{\lambda}{\tau}-l-1\right) G_{1}\left(R^{N-l}-R^{N+l}\right)\right. \\
& \left.+\left(\frac{\lambda}{\tau}-l\right) G_{1}\left(R^{N-l-1}-R^{N+l+1}\right)\right) \\
& \times\left(\psi^{h}(x)-\varphi^{h}(x)\right),
\end{aligned}
$$

for difference scheme (25), and on the following theorem on the coercivity inequality for the solution of the elliptic difference problem in $L_{2 h}$.

Theorem 8 (see [28]). For the solution of the elliptic difference problem

$$
\begin{gathered}
A_{h}^{x} u^{h}(x)=\omega^{h}(x), \quad x \in \widetilde{\Omega}_{h}, \\
D^{h} u^{h}(x)=0, \quad x \in S_{h},
\end{gathered}
$$

the following coercivity inequality holds:

$$
\sum_{r=1}^{n}\left\|\left(u_{k}^{h}\right)_{\bar{x}_{r} \bar{x}_{r}, j_{r}}\right\|_{L_{2 h}} \leq M\left\|\omega^{h}\right\|_{L_{2 h}},
$$

where $M$ does not depend on $h$ and $\omega^{h}$.

\section{Numerical Results}

We have not been able to obtain a sharp estimate for the constants figuring in the stability estimates. Therefore, we will give the following results of numerical experiments of the inverse problem for the two-dimensional elliptic equation with Dirichlet-Neumann boundary conditions

$$
\begin{aligned}
& -\frac{\partial^{2} u(t, x)}{\partial t^{2}}-\frac{\partial}{\partial x}\left((2+\cos x) \frac{\partial u(t, x)}{\partial x}\right)+u(t, x) \\
& =f(t, x)+p(x), \quad 0<x<\pi, 0<t<T, \\
& f(t, x)=-\exp (-t) \cos (x) \\
& +(\exp (-t)+t)(3 \cos (x)+\cos (2 x)), \\
& u(0, x)=2 \cos (x), \quad 0 \leq x \leq \pi, \\
& u(T, x)=(\exp (-T)+T+1) \cos (x), \quad 0 \leq x \leq \pi, \\
& u(\lambda, x)=(\exp (-\lambda)+\lambda+1) \cos (x), \quad 0 \leq x \leq \pi, \\
& u_{x}(t, 0)=u_{x}(t, \pi)=0, \quad 0 \leq t \leq T, \lambda=\frac{3 T}{5} .
\end{aligned}
$$

It is clear that $u(t, x)=(\exp (-t)+t+1) \cos (x)$ and $p(x)=$ $\sin (x)+(x+2) \cos (x)$ are the exact solutions of (34).
We can obtain $u(t, x)$ by formula $u(t, x)=v(t, x)+w(t, x)$, where $v(t, x)$ is the solution of the nonlocal boundary value problem

$$
\begin{aligned}
& -\frac{d^{2} v(t, x)}{d t^{2}}-\frac{\partial}{\partial x}\left((2+\cos x) \frac{\partial v(t, x)}{\partial x}\right)+v(t, x) \\
& =f(t, x), \quad 0<x<\pi, 0<t<T, \\
& v(0, x)-v(\lambda, x)=(1-\exp (-\lambda)-\lambda) \cos (x), \\
& 0 \leq x \leq \pi \\
& v(T, x)-v(\lambda, x) \\
& =(\exp (-T)-\exp (-\lambda)+T-\lambda) \cos (x), \\
& 0 \leq x \leq \pi, \\
& v_{x}(t, 0)=v_{x}(t, \pi)=0, \quad 0 \leq t \leq T,
\end{aligned}
$$

and $w(t, x)$ is the solution of the boundary value problem

$$
\begin{gathered}
-\frac{d^{2} w(t, x)}{d t^{2}}-\frac{\partial}{\partial x}\left((2+\cos x) \frac{\partial w(t, x)}{\partial x}\right)+w(t, x) \\
=p(x), \quad 0<x<\pi, 0<t<T, \\
w(0, x)=(\exp (-\lambda)+\lambda+1) \cos (x)-v(\lambda, x), \\
0 \leq x \leq \pi, \\
w(T, x)=(\exp (-\lambda)+\lambda+1) \cos (x)-v(\lambda, x), \\
\quad 0 \leq x \leq \pi, \\
w_{x}(t, 0)=w_{x}(t, \pi)=0, \quad 0 \leq t \leq T .
\end{gathered}
$$

Introduce small parameters $\tau$ and $h$ such that $N \tau=$ $T, M h=\pi$. For approximate solution of nonlocal boundary value problem (35) we consider the set $[0, T]_{\tau} \times[0, \pi]_{h}$ of a family of grid points

$$
\begin{aligned}
& {[0, T]_{\tau} \times[0, \pi]_{h}} \\
& \quad=\left\{\left(t_{k}, x_{n}\right): t_{k}=k \tau\right. \\
& \left.\quad k=0, \ldots, N, x_{n}=n h, n=0, \ldots, M\right\} .
\end{aligned}
$$


Applying (21), we obtain difference schemes of the first order of accuracy in $t$ and the second order of accuracy in $x$

$$
\begin{gathered}
\frac{v_{n}^{k+1}-2 v_{n}^{k}+v_{n}^{k-1}}{\tau^{2}}+\left(2+\cos \left(x_{n}\right)\right) \frac{v_{n+1}^{k}-2 v_{n}^{k}+v_{n-1}^{k}}{h^{2}} \\
-\sin \left(x_{n}\right) \frac{v_{n+1}^{k}-v_{n-1}^{k}}{2 h}-v_{n}^{k}=\theta_{n}^{k}, \\
\theta_{n}^{k}=-f\left(t_{k}, x_{n}\right), \quad k=1, \ldots, N-1, n=1, \ldots, M-1, \\
v_{0}^{k}-v_{1}^{k}=v_{M}^{k}-v_{M-1}^{k}=0, \quad k=0, \ldots, N, \\
v_{n}^{0}-v_{n}^{l}=(1-\exp (-\lambda)-\lambda) \cos \left(x_{n}\right), \quad n=0, \ldots, M, \\
v_{n}^{N}-v_{n}^{l}=\left(\exp \left(-t_{N}\right)-\exp (-\lambda)+t_{N}-\lambda\right) \cos \left(x_{n}\right), \\
n=0, \ldots, M, \quad l=\left[\frac{\lambda}{\tau}\right],
\end{gathered}
$$

for the approximate solutions of the nonlocal boundary value problem (35), and

$$
\begin{gathered}
\frac{w_{n}^{k+1}-2 w_{n}^{k}+w_{n}^{k-1}}{\tau^{2}}+\left(2+\cos \left(x_{n}\right)\right) \frac{w_{n+1}^{k}-2 w_{n}^{k}+w_{n-1}^{k}}{h^{2}} \\
-\sin \left(x_{n}\right) \frac{w_{n+1}^{k}-w_{n-1}^{k}}{2 h}-w_{n}^{k} \\
=-p_{n}, \quad k=1, \ldots, N-1, \\
p_{n}=p\left(x_{n}\right), n=1, \ldots, M-1, \\
w_{0}^{k}-w_{1}^{k}=w_{M}^{k}-w_{M-1}^{k}=0, \quad k=0, \ldots, N, \\
w_{n}^{0}=(\exp (-\lambda)+\lambda+1) \cos \left(x_{n}\right)-v_{n}^{l}, \\
n=0, \ldots, M, l=\left[\frac{\lambda}{\tau}\right], \\
w_{n}^{N}=(\exp (-\lambda)+\lambda+1) \cos \left(x_{n}\right)-v_{n}^{l}, \\
n=0, \ldots, M,
\end{gathered}
$$

for the approximate solutions of the boundary value problem (36).

By using (22) and second order of accuracy in $x$ approximation of $A$, we get the following values of $p$ in grid points:

$$
\begin{aligned}
p_{n}= & -\frac{\left(2+\cos \left(x_{n}\right)\right)}{h^{2}}\left(\left(\varphi_{n+1}-v_{n+1}^{0}\right)-2\left(\varphi_{n}-v_{n}^{0}\right)\right. \\
& \left.+\left(\varphi_{n-1}-v_{n-1}^{0}\right)\right)+\frac{\sin \left(x_{n}\right)}{2 h} \\
& \times\left(\left(\varphi_{n+1}-v_{n+1}^{0}\right)-\left(\varphi_{n-1}-v_{n-1}^{0}\right)\right) \\
& +\left(\varphi_{n}-v_{n}^{0}\right), \quad n=1, \ldots, M-1 .
\end{aligned}
$$

We can rewrite difference scheme (38) in the matrix form

$$
\begin{gathered}
A_{n} v_{n+1}+B_{n} v_{n}+C_{n} v_{n-1}=I \theta_{n}^{k}, \quad n=1, \ldots, M-1, \\
v_{0}=v_{1}, \quad v_{M}=v_{M-1} .
\end{gathered}
$$

Here, $I$ is the $(N+1) \times(N+1)$ identity matrix, $A_{n}, B_{n}, C_{n}$ are $(N+1) \times(N+1)$ square matrices, and $\theta_{n}$ is a $(N+1) \times 1$ column matrix which are defined by

$$
A_{n}=\left[\begin{array}{ccccccccc}
0 & 0 & 0 & 0 & \cdots & 0 & 0 & 0 & 0 \\
0 & a_{n} & 0 & 0 & \cdots & 0 & 0 & 0 & 0 \\
0 & 0 & a_{n} & 0 & \cdots & 0 & 0 & 0 & 0 \\
0 & 0 & 0 & a_{n} & \cdots & 0 & 0 & 0 & 0 \\
\vdots & \vdots & \vdots & \vdots & \cdots & \vdots & \vdots & \vdots & \vdots \\
0 & 0 & 0 & 0 & \cdots & a_{n} & 0 & 0 & 0 \\
0 & 0 & 0 & 0 & \cdots & 0 & a_{n} & 0 & 0 \\
0 & 0 & 0 & 0 & \cdots & 0 & 0 & a_{n} & 0 \\
0 & 0 & 0 & 0 & \cdots & 0 & 0 & 0 & 0
\end{array}\right],
$$




$$
\begin{gathered}
\theta_{n}=\left[\begin{array}{c}
\theta_{n}^{0} \\
\vdots \\
\theta_{n}^{N}
\end{array}\right], \\
\theta_{n}^{0}=(1-\exp (-\lambda)-\lambda) \cos \left(x_{n}\right), \\
\theta_{n}^{N}=\left(\exp \left(-t_{N}\right)-\exp (-\lambda)+t_{N}-\lambda\right) \cos \left(x_{n}\right), \\
\theta_{n}^{k}=-f\left(t_{k}, x_{n}\right), \quad k=1, \ldots, M-1, N-1, n=1, \ldots, M-1, \\
v_{s}=\left[\begin{array}{c}
v_{s}^{0} \\
\vdots \\
v_{s}^{N}
\end{array}\right]_{(N+1) \times 1} \quad s=n-1, n, n+1 .
\end{gathered}
$$

For solving (41) we use the modified Gauss elimination method (see [29]). Namely, we seek solution of (41) by the formula

$$
v_{n}=\alpha_{n+1} v_{n+1}+\beta_{n+1}, \quad n=M-1, \ldots, 1,
$$

where $v_{M}=\overrightarrow{0}, \alpha_{n}(n=1, \ldots, M-1)$ are $(N+1) \times(N+1)$ square matrices and $\beta_{n}(n=1, \ldots, M-1)$ are $(N+1) \times 1$ column matrices. For $\alpha_{n+1}, \beta_{n+1}$, we get formulas

$$
\begin{gathered}
\alpha_{n+1}=-\left(B_{n}+C_{n} \alpha_{n}\right)^{-1} A_{n} \\
\beta_{n+1}=-\left(B_{n}+C_{n} \alpha_{n}\right)^{-1}\left(I \theta_{n}-C_{n} \beta_{n}\right), \quad n=1, \ldots, M-1,
\end{gathered}
$$

where $\alpha_{1}$ is the $(N+1) \times(N+1)$ identity matrix and $\beta_{1}$ is the $(N+1) \times 1$ zero column vector. form

Futher, we rewrite difference scheme (39) in the matrix

$$
\begin{gathered}
A_{n} w_{n+1}+E_{n} w_{n}+C_{n} w_{n-1}=I \eta_{n}^{k}, \\
n=1, \ldots, M-1, \\
w_{0}=w_{1}, \quad w_{M}=w_{M-1} .
\end{gathered}
$$

Here,

$$
E_{n}=\left[\begin{array}{ccccccccc}
1 & 0 & 0 & 0 & \cdots & 0 & 0 & 0 & 0 \\
d & b_{n} & d & 0 & \cdots & 0 & 0 & 0 & 0 \\
0 & d & b_{n} & d & \cdots & 0 & 0 & 0 & 0 \\
0 & 0 & d & b_{n} & \cdots & 0 & 0 & 0 & 0 \\
\vdots & \vdots & \vdots & \vdots & \ddots & \vdots & \vdots & \vdots & \vdots \\
0 & 0 & 0 & 0 & \cdots & b_{n} & d & 0 & 0 \\
0 & 0 & 0 & 0 & \cdots & d & b_{n} & d & 0 \\
0 & 0 & 0 & 0 & \cdots & 0 & d & b_{n} & d \\
0 & 0 & 0 & 0 & \cdots & 0 & 0 & 0 & 1
\end{array}\right],
$$

$A_{n}$ and $C_{n}$ are defined by (42) and (43) and $(N+1) \times 1$ column matrix $\eta_{n}$ is defined by

$$
\begin{gathered}
\eta_{n}=\left[\begin{array}{c}
\eta_{n}^{0} \\
\vdots \\
\eta_{n}^{N}
\end{array}\right], \\
\eta_{n}^{0}=(\exp (-\lambda)+\lambda+1) \cos \left(x_{n}\right)-v_{n}^{l}, \\
\eta_{n}^{N}=(\exp (-\lambda)+\lambda+1) \cos \left(x_{n}\right)-v_{n}^{l}, \quad n=1, \ldots, M-1, \\
\eta_{n}^{k}=-p_{n}, \quad k=1, \ldots, N-1, n=1, \ldots, M-1, \\
w_{s}=\left[\begin{array}{c}
w_{s}^{0} \\
\vdots \\
w_{s}^{N}
\end{array}\right]_{(N+1) \times 1}, \quad s=n-1, n, n+1 .
\end{gathered}
$$

Now we present second order of accuracy in $t$ and $x$ difference schemes for problems (35) and (36). Applying (27) and formulas for sufficiently smooth function $\rho$

$$
\begin{gathered}
\frac{\rho\left(x_{n+1}\right)-\rho\left(x_{n-1}\right)}{2 h}-\rho^{\prime}\left(x_{n}\right)=O\left(h^{2}\right), \\
\frac{\rho\left(x_{n+1}\right)-2 \rho\left(x_{n}\right)+\rho\left(x_{n-1}\right)}{h^{2}}-\rho^{\prime \prime}\left(x_{n}\right)=O\left(h^{2}\right), \\
\frac{10 \rho(0)-15 \rho(h)+6 \rho(2 h)-\rho(3 h)}{h^{3}}-\rho^{\prime \prime \prime}(0)=O\left(h^{2}\right), \\
\frac{-3 \rho(0)+4 \rho(h)-\rho(2 h)}{2 h}-\rho^{\prime}(0)=O\left(h^{2}\right), \\
\frac{10 \rho(\pi)-15 \rho(\pi-h)+6 \rho(\pi-2 h)-\rho(\pi-3 h)}{h^{3}} \\
\frac{-3 \rho(\pi)+4 \rho(\pi-h)-\rho(\pi-2 h)}{2 h}-\rho^{\prime}(\pi)=O\left(h^{2}\right),
\end{gathered}
$$

we get

$$
\begin{aligned}
& \frac{v_{n}^{k+1}-2 v_{n}^{k}+v_{n}^{k-1}}{\tau^{2}}+\left(2+\cos \left(x_{n}\right)\right) \frac{v_{n+1}^{k}-2 v_{n}^{k}+v_{n-1}^{k}}{h^{2}} \\
& -\sin \left(x_{n}\right) \frac{v_{n+1}^{k}-v_{n-1}^{k}}{2 h}-v_{n}^{k}=\theta_{n}^{k}
\end{aligned}
$$




$$
\begin{gathered}
\theta_{n}^{k}=-f\left(t_{k}, x_{n}\right), \quad k=1, \ldots, N-1, \quad n=1, \ldots, M-1, \\
-3 v_{0}^{k}+4 v_{1}^{k}-v_{2}^{k} \\
=-3 v_{M}^{k}+4 v_{M-1}^{k}-v_{M-2}^{k}=0, \quad k=0, \ldots, N \\
10 v_{0}^{k}-15 v_{1}^{k}+6 v_{2}^{k}-v_{3}^{k} \\
=10 v_{M}^{k}-15 v_{M-1}^{k}+6 v_{M-2}^{k}-v_{M-3}^{k}=0, \\
v_{n}^{0}+\left(\frac{\lambda}{\tau}-l-1\right) v_{n}^{l}-\left(\frac{\lambda}{\tau}-l\right) v_{n}^{l+1} \\
=(1-\exp (-\lambda)-\lambda) \cos \left(x_{n}\right), \quad n=0, \ldots, M \\
v_{n}^{N}+\left(\frac{\lambda}{\tau}-l-1\right) v_{n}^{l}-\left(\frac{\lambda}{\tau}-l\right) v_{n}^{l+1} \\
=\left(\exp \left(-t_{N}\right)-\exp (-\lambda)+t_{N}-\lambda\right) \cos \left(x_{n}\right) \\
n=0, \ldots, M
\end{gathered}
$$

difference scheme for nonlocal problem (35), and

$$
\begin{aligned}
& \frac{w_{n}^{k+1}-2 w_{n}^{k}+w_{n}^{k-1}}{\tau^{2}}+\left(2+\cos \left(x_{n}\right)\right) \frac{w_{n+1}^{k}-2 w_{n}^{k}+w_{n-1}^{k}}{h^{2}} \\
& -\sin \left(x_{n}\right) \frac{w_{n+1}^{k}-w_{n-1}^{k}}{2 h}=-p_{n} \\
& k=1, \ldots, N-1, \quad p_{n}=p\left(x_{n}\right), \quad n=1, \ldots, M-1, \\
& -3 w_{0}^{k}+4 w_{1}^{k}-w_{2}^{k}=-3 w_{M}^{k}+4 w_{M-1}^{k}-w_{M-2}^{k}=0, \\
& k=0, \ldots, N \text {, } \\
& 10 w_{0}^{k}-15 w_{1}^{k}+6 w_{2}^{k}-w_{3}^{k} \\
& =10 w_{M}^{k}-15 w_{M-1}^{k}+6 w_{M-2}^{k}-w_{M-3}^{k}=0, \\
& w_{n}^{0}=(\exp (-\lambda)+\lambda+1) \cos \left(x_{n}\right) \\
& +\left(\frac{\lambda}{\tau}-l-1\right) v_{n}^{l}-\left(\frac{\lambda}{\tau}-l\right) v_{n}^{l+1}, \quad n=0, \ldots, M, \\
& w_{n}^{N}=(\exp (-\lambda)+\lambda+1) \cos \left(x_{n}\right) \\
& +\left(\frac{\lambda}{\tau}-l-1\right) v_{n}^{l}-\left(\frac{\lambda}{\tau}-l\right) v_{n}^{l+1} \\
& \xi_{n}=\xi\left(x_{n}\right), \quad n=0, \ldots, M,
\end{aligned}
$$

difference scheme for boundary value problem (36).
By difference scheme (52), we write in matrix form

$$
\begin{gathered}
A_{n} v_{n+1}+B_{n} v_{n}+C_{n} v_{n-1}=I \theta_{n}^{k}, \quad n=1, \ldots, M-1, \\
-3 v_{0}+4 v_{1}-v_{2}=0 \\
-3 v_{M}+4 v_{M-1}-v_{M-2}=0
\end{gathered}
$$

where $A_{n}, C_{n}$ are defined by (42), (43), (44), and $B_{n}$ is defined by

$$
\begin{aligned}
& B_{n}=\left[\begin{array}{cccccccccccccc}
1 & 0 & 0 & 0 & \cdots & 0 & y & z & 0 & \cdots & 0 & 0 & 0 & 0 \\
d & b_{n} & d & 0 & \cdots & 0 & 0 & 0 & 0 & \cdots & 0 & 0 & 0 & 0 \\
0 & d & b_{n} & d & \cdots & 0 & 0 & 0 & 0 & \cdots & 0 & 0 & 0 & 0 \\
0 & 0 & d & b_{n} & \cdots & 0 & 0 & 0 & 0 & \cdots & 0 & 0 & 0 & 0 \\
\vdots & \vdots & \vdots & \vdots & \vdots & \vdots & \vdots & \vdots & \vdots & \vdots & \vdots & \vdots & \vdots & \vdots \\
0 & 0 & 0 & 0 & \cdots & 0 & 0 & 0 & 0 & \cdots & b_{n} & d & 0 & 0 \\
0 & 0 & 0 & 0 & \cdots & 0 & 0 & 0 & 0 & \cdots & d & b_{n} & d & 0 \\
0 & 0 & 0 & 0 & \cdots & 0 & 0 & 0 & 0 & \cdots & 0 & d & b_{n} & d \\
0 & 0 & 0 & 0 & \cdots & 0 & y & z & 0 & \cdots & 0 & 0 & 0 & 1
\end{array}\right], \\
& d=\frac{1}{\tau^{2},} y=\left(\frac{\lambda}{\tau}-l-1\right), b^{2}=-\left(\frac{\lambda}{\tau}-l\right) .
\end{aligned}
$$

We seek solution of (54) by the formula

$$
v_{n}=\alpha_{n} v_{n+1}+\beta_{n} v_{n+2}+\gamma_{n}, \quad n=M-2, \ldots, 0,
$$

where $\alpha_{n}, \beta_{n}(n=0, \ldots, M-2)$ are $(N+1) \times(N+1)$ square matrices and $\gamma_{n}(n=0, \ldots, M-2)$ are $(N+1) \times 1$ column matrices. For the solution of difference equation (41) we need to use the following formulas for $\alpha_{n}, \beta_{n}$ :

$$
\begin{gathered}
\alpha_{n}=-\left(B_{n}+C_{n} \alpha_{n-1}\right)^{-1}\left(A_{n}+C_{n} \beta_{n-1}\right), \\
\beta_{n}=0, \\
\gamma_{n}=-\left(B_{n}+C_{n} \alpha_{n-1}\right)^{-1}\left(I \theta_{n}-C_{n} \gamma_{n-1}\right), \quad n=1, \ldots, M-1,
\end{gathered}
$$

where

$$
\begin{array}{ll}
\alpha_{0}=\frac{4}{3} I, & \beta_{0}=-\frac{1}{3} I, \\
\alpha_{1}=\frac{8}{5} I, & \beta_{1}=-\frac{3}{5} I, \\
\alpha_{M-2}=4 I, & \beta_{M-2}=-3 I, \\
\alpha_{M-3}=\frac{8}{3} I, & \beta_{M-3}=-\frac{5}{3} I,
\end{array}
$$

and $\gamma_{0}, \gamma_{1}, \gamma_{M-2}, \gamma_{M-3}$ are the $(N+1) \times 1$ zero column vector. For $v_{M}$ and $v_{M-1}$ we have

$$
\begin{gathered}
v_{M}=\left(Q_{11}-Q_{12} Q_{22}^{-1} Q_{21}\right)^{-1}\left(G_{1}-Q_{12} Q_{22}^{-1} G_{2}\right), \\
v_{M-1}=Q_{22}^{-1}\left(G_{2}-Q_{21} v_{M}\right),
\end{gathered}
$$


where

$$
\begin{gathered}
Q_{11}=-3 A_{M-2}-8 B_{M-2}-8 C_{M-2} \alpha_{M-3}-3 C_{M-2} \beta_{M-3} \\
Q_{12}=4 A_{M-2}+9 B_{M-2}+9 C_{M-2} \alpha_{M-3}+4 C_{M-2} \beta_{M-3} \\
Q_{21}=-3 B_{M-1}-8 C_{M-1} \\
Q_{22}=A_{M-1}+4 B_{M-1}+9 C_{M-1} \\
G_{1}=I \theta_{M-2}-C_{M-2} \gamma_{M-3}, \quad G_{2}=I \theta_{M-1} .
\end{gathered}
$$

We can rewrite difference scheme (53) in the matrix form

$$
\begin{gathered}
A_{n} w_{n+1}+E_{n} w_{n}+C_{n} w_{n-1}=I \eta_{n}^{k}, \quad n=1, \ldots, M-1, \\
-3 w_{0}+4 w_{1}-w_{2}=0 \\
-3 w_{M}+4 w_{M-1}-w_{M-2}=0
\end{gathered}
$$

where $A_{n}, E_{n}, C_{n}$ are defined by (42), (49), (43), and (44) and $\eta_{n}$ is defined by

$$
\begin{gathered}
\eta_{n}=\left[\begin{array}{c}
\eta_{n}^{0} \\
\vdots \\
\eta_{n}^{N}
\end{array}\right], \\
\eta_{n}^{0}=(\exp (-\lambda)+\lambda+1) \cos \left(x_{n}\right) \\
+\left(\frac{\lambda}{\tau}-l-1\right) v_{n}^{l}-\left(\frac{\lambda}{\tau}-l\right) v_{n}^{l+1}, \\
\eta_{n}^{N}=(\exp (-\lambda)+\lambda+1) \cos \left(x_{n}\right)+\left(\frac{\lambda}{\tau}-l-1\right) v_{n}^{l} \\
-\left(\frac{\lambda}{\tau}-l\right) v_{n}^{l+1}, \quad n=0, \ldots, M, \\
\eta_{n}^{k}=-p_{n}, \quad k=1, \ldots, N-1, \quad n=1, \ldots, M-1 .
\end{gathered}
$$

Now, we give the results of the numerical realization of finite difference method for (34) by using MATLAB programs. The numerical solutions are recorded for $T=$ 2 and different values of $N=M$. Grid functions $v_{n}^{k}, u_{n}^{k}$ represent the numerical solutions of difference schemes for auxiliary nonlocal problem (35) and inverse problem (34) at $\left(t_{k}, x_{n}\right)$, respectively. Grid function $p_{n}$ calculated by (40) represents numerical solution at $x_{n}$ for unknown function $p$. The errors are computed by the norms

$$
\begin{gathered}
E v_{M}^{N}=\max _{1 \leq k \leq N-1}\left(\sum_{n=1}^{M-1}\left|v\left(t_{k}, x_{n}\right)-v_{n}^{k}\right|^{2} h\right)^{1 / 2}, \\
E u_{M}^{N}=\max _{1 \leq k \leq N-1}\left(\sum_{n=1}^{M-1}\left|u\left(t_{k}, x_{n}\right)-u_{n}^{k}\right|^{2} h\right)^{1 / 2}, \\
E p_{M}=\left(\sum_{n=1}^{M-1}\left|p\left(x_{n}\right)-p_{n}\right|^{2} h\right)^{1 / 2} .
\end{gathered}
$$

TABLE 1: Error analysis for nonlocal problem.

\begin{tabular}{lcccc}
\hline & $N=M=$ & $N=M=$ & $N=M=$ & $N=M=$ \\
20 & 40 & 80 & 160 \\
\hline $\begin{array}{l}\text { Difference } \\
\text { scheme (38) }\end{array}$ & 0.30522 & 0.14933 & 0.073953 & 0.036814 \\
$\begin{array}{l}\text { Difference } \\
\text { scheme (52) }\end{array}$ & 0.024714 & 0.0031054 & $4.36 \times 10^{-4}$ & $7.52 \times 10^{-5}$ \\
\hline
\end{tabular}

TABLE 2: Error analysis for $p$.

\begin{tabular}{lcccc}
\hline & $\begin{array}{c}N=M= \\
20\end{array}$ & $\begin{array}{c}N=M= \\
40\end{array}$ & $\begin{array}{c}N=M= \\
80\end{array}$ & $\begin{array}{c}N=M= \\
160\end{array}$ \\
\hline $\begin{array}{l}\text { Difference } \\
\text { scheme (38), } \\
(40)\end{array}$ & 0.57878 & 0.33755 & 0.20387 & 0.12905 \\
$\begin{array}{l}\text { Difference } \\
\text { scheme (52), } \\
(40)\end{array}$ & 0.058201 & 0.010646 & 0.0020228 & $4.03 \times 10^{-4}$ \\
\hline
\end{tabular}

TABLE 3: Error analysis for $u$.

\begin{tabular}{lcccc}
\hline & $\begin{array}{c}N=M= \\
20\end{array}$ & $\begin{array}{c}N=M= \\
40\end{array}$ & $\begin{array}{c}N=M= \\
80\end{array}$ & $\begin{array}{c}N=M= \\
160\end{array}$ \\
\hline $\begin{array}{l}\text { Difference } \\
\text { scheme (38), } \\
(40),(39)\end{array}$ & 0.088225 & 0.038586 & 0.01815 & 0.008818 \\
$\begin{array}{l}\text { Difference } \\
\text { scheme (52), } \\
(40),(53)\end{array}$ & 0.017034 & 0.0020225 & $2.47 \times 10^{-4}$ & $3.08 \times 10^{-5}$ \\
\hline
\end{tabular}

Tables 1-3 present the error between the exact solution and numerical solutions derived by corresponding difference schemes. The results are recorded for $N=M=20,40,80$ and 160, respectively. The tables show that the second order of accuracy difference scheme is more accurate than the first order of accuracy difference scheme for both auxiliary nonlocal and inverse problems. Table 1 contains error between the exact and approximate solutions $v$ of auxiliary nonlocal boundary value problem (35). Table 2 includes error between the exact and approximate solutions $p$ of inverse problem (34). Table 3 represents error between the exact solution $u$ of inverse problem (34) and approximate solution which is derived by the first and second orders accuracy of difference schemes.

\section{Conclusion}

In this paper, inverse problem for multidimensional elliptic equation with Dirichlet-Neumann conditions is considered. The stability and coercive stability estimates for solution of this problem are established. First and second order of accuracy difference schemes are presented for approximate solutions of inverse problem. Theorems on the stability and coercive stability inequalities for difference schemes are proved. The theoretical statements for the solution of these difference schemes are supported by the results of numerical example in a two-dimensional case. As it can be seen from Tables 1-3, second order of accuracy difference 
scheme is more accurate compared with the first order of accuracy difference scheme. Moreover, applying the result of the monograph [29] the high order of accuracy difference schemes for the numerical solution of the boundary value problem (2) can be presented.

\section{Acknowledgment}

The authors would like to thank Professor Dr. Allaberen Ashyralyev (Fatih University, Turkey) for his helpful suggestions aimed at the improvement of the present paper.

\section{References}

[1] A. I. Prilepko, D. G. Orlovsky, and I. A. Vasin, Methods for Solving Inverse Problems in Mathematical Physics, vol. 231 of Monographs and Textbooks in Pure and Applied Mathematics, Marcel Dekker, New York, NY, USA, 2000.

[2] A. A. Samarskii and P. N. Vabishchevich, Numerical Methods for Solving Inverse Problems of Mathematical Physics, Inverse and Ill-posed Problems Series, Walter de Gruyter GmbH \& Company, Berlin, Germany, 2007.

[3] V. V. Soloviev, "Inverse problems of source determination for the Poisson equation on the plane," Zhurnal Vychislitel'noi Matematiki i Matematicheskoi Fiziki, vol. 44, no. 5, pp. 862-871, 2004 (Russian).

[4] D. G. Orlovskiî, "Inverse Dirichlet problem for an equation of elliptic type," Differential Equations, vol. 44, no. 1, pp. 124-134, 2008.

[5] D. Orlovsky and S. Piskarev, "On approximation of inverse problems for abstract elliptic problems," Journal of Inverse and Ill-Posed Problems, vol. 17, no. 8, pp. 765-782, 2009.

[6] A. Ashyralyev and A. S. Erdogan, "Well-posedness of the inverse problem of a multidimensional parabolic equation," Vestnik of Odessa National University, Mathematics and Mechanics, vol. 15, no. 18, pp. 129-135, 2010.

[7] A. Ashyralyev, "On the problem of determining the parameter of a parabolic equation," Ukrainian Mathematical Journal, vol. 62, no. 9, pp. 1397-1408, 2011.

[8] A. Ashyralyev and A. S. Erdoğan, "On the numerical solution of a parabolic inverse problem with the Dirichlet condition," International Journal of Mathematics and Computation, vol. 11, no. J11, pp. 73-81, 2011.

[9] C. Ashyralyyev, A. Dural, and Y. Sozen, "Finite difference method for the reverse parabolic problem," Abstract and Applied Analysis, vol. 2012, Article ID 294154, 17 pages, 2012.

[10] C. Ashyralyyev and O. Demirdag, "The difference problem of obtaining the parameter of a parabolic equation," Abstract and Applied Analysis, vol. 2012, Article ID 603018, 14 pages, 2012.

[11] Ch. Ashyralyyev and M. Dedeturk, "A finite difference method for the inverse elliptic problem with the Dirichlet condition," Contemporary Analysis and Applied Mathematics, vol. 1, no. 2, pp. 132-155, 2013.

[12] A. Ashyralyev and M. Urun, "Determination of a control parameter for the Schrodinger equation," Contemporary Analysis and Applied Mathematics, vol. 1, no. 2, pp. 156-166, 2013.

[13] M. Dehghan, "Determination of a control parameter in the twodimensional diffusion equation," Applied Numerical Mathematics, vol. 37, no. 4, pp. 489-502, 2001.
[14] V. G. Romanov, "A three-dimensional inverse problem of viscoelasticity," Doklady Mathematics, vol. 84, no. 3, pp. 833836, 2011.

[15] S. I. Kabanikhin, "Definitions and examples of inverse and illposed problems," Journal of Inverse and Ill-Posed Problems, vol. 16 , no. 4, pp. 317-357, 2008.

[16] Y. S. Éidel'man, "An inverse problem for an evolution equation," Mathematical Notes, vol. 49, no. 5, pp. 535-540, 1991.

[17] K. Sakamoto and M. Yamamoto, "Inverse heat source problem from time distributing overdetermination," Applicable Analysis, vol. 88, no. 5, pp. 735-748, 2009.

[18] A. V. Gulin, N. I. Ionkin, and V. A. Morozova, "On the stability of a nonlocal two-dimensional finite-difference problem," Differential Equations, vol. 37, no. 7, pp. 970-978, 2001.

[19] G. Berikelashvili, "On a nonlocal boundary-value problem for two-dimensional elliptic equation," Computational Methods in Applied Mathematics, vol. 3, no. 1, pp. 35-44, 2003.

[20] M. P. Sapagovas, "Difference method of increased order of accuracy for the Poisson equation with nonlocal conditions," Differential Equations, vol. 44, no. 7, pp. 1018-1028, 2008.

[21] A. Ashyralyev, "A note on the Bitsadze-Samarskii type nonlocal boundary value problem in a Banach space," Journal of Mathematical Analysis and Applications, vol. 344, no. 1, pp. 557-573, 2008.

[22] D. Orlovsky and S. Piskarev, "The approximation of BitzadzeSamarsky type inverse problem for elliptic equations with Neumann conditions," Contemporary Analysis and Applied Mathematics, vol. 1, no. 2, pp. 118-131, 2013.

[23] A. Ashyralyev and F. S. O. Tetikoglu, "FDM for elliptic equations with Bitsadze-Samarskii-Dirichlet conditions," Abstract and Applied Analysis, vol. 2012, Article ID 454831, 22 pages, 2012.

[24] A. Ashyralyev and E. Ozturk, "The numerical solution of the Bitsadze-Samarskii nonlocal boundary value problems with the Dirichlet-Neumann condition," Abstract and Applied Analysis, vol. 2013, Article ID 730804, 13 pages, 2012.

[25] A. Ashyralyev, "Well-posedness of the difference schemes for elliptic equations in $C_{\tau}^{\beta, \gamma}(E)$ spaces," Applied Mathematics Letters, vol. 22, no. 3, pp. 390-395, 2009.

[26] S. G. Krein, Linear Differential Equations in Banach Space, Nauka, Moscow, Russia, 1966.

[27] A. Ashyralyev and P. E. Sobolevskii, New Difference Schemes for Partial Differential Equations, vol. 148 of Operator Theory: Advances and Applications, Birkhäuser, Basel, Switzerland, 2004.

[28] P. E. Sobolevskii, Difference Methods for the Approximate Solution of Differential Equations, Voronezh State University Press, Voronezh, Russia, 1975.

[29] A. A. Samarskii and E. S. Nikolaev, Numerical Methods for Grid Equations, vol. 2, Birkhäuser, Basel, Switzerland, 1989. 


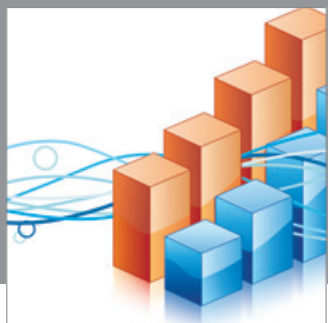

Advances in

Operations Research

mansans

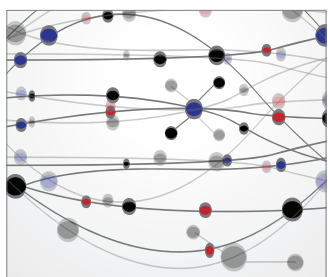

The Scientific World Journal
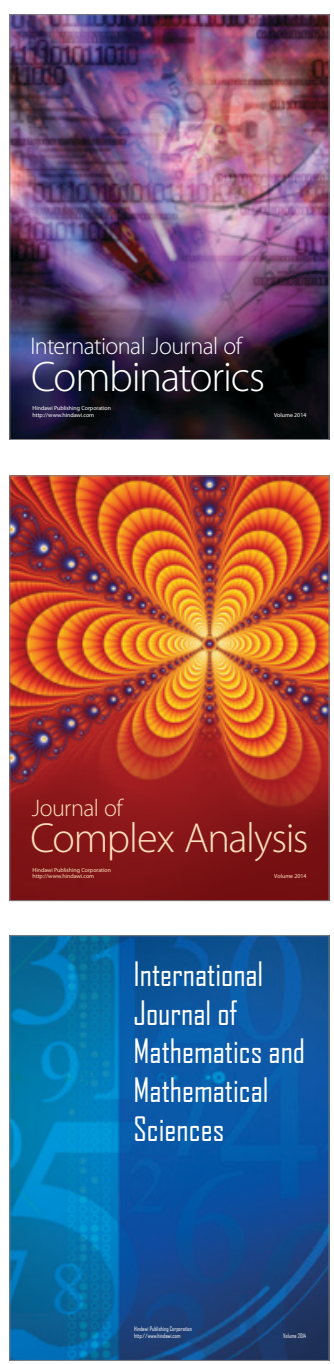
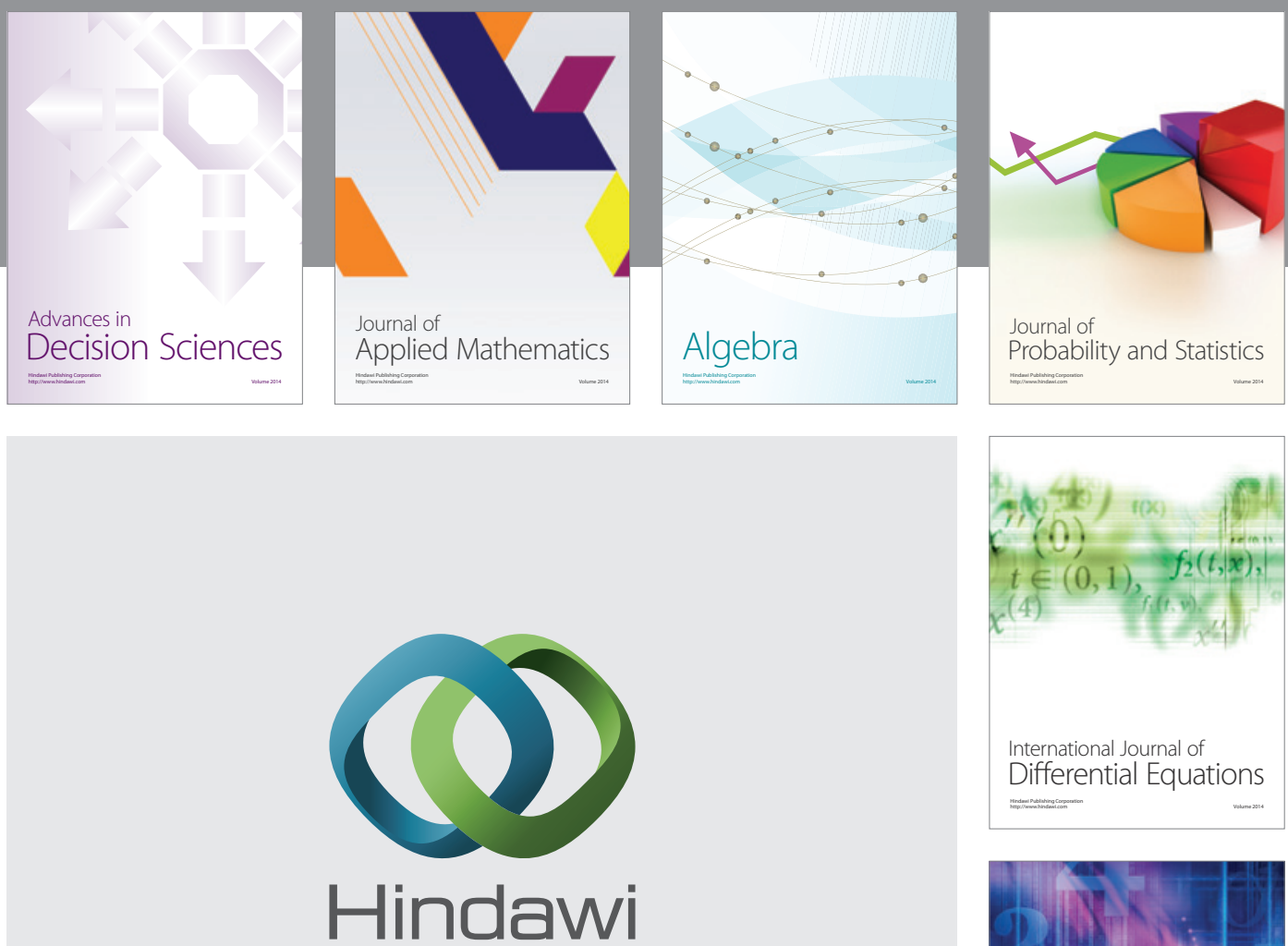

Submit your manuscripts at http://www.hindawi.com
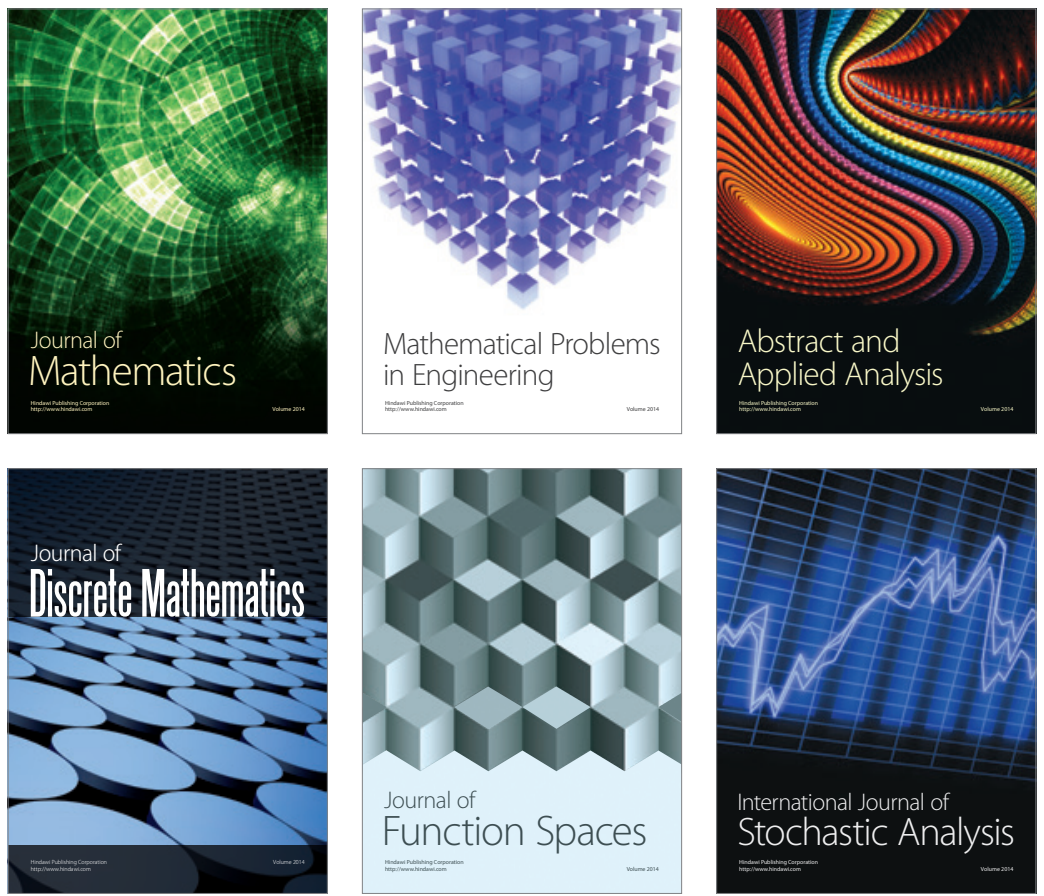

Journal of

Function Spaces

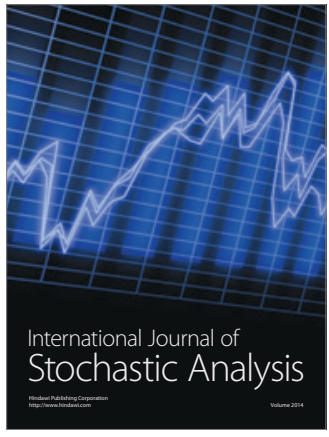

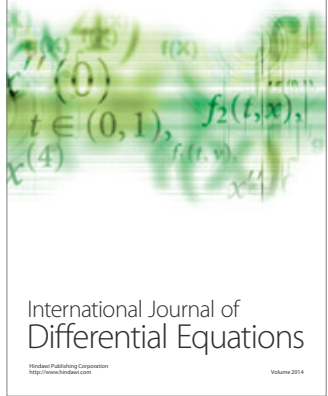
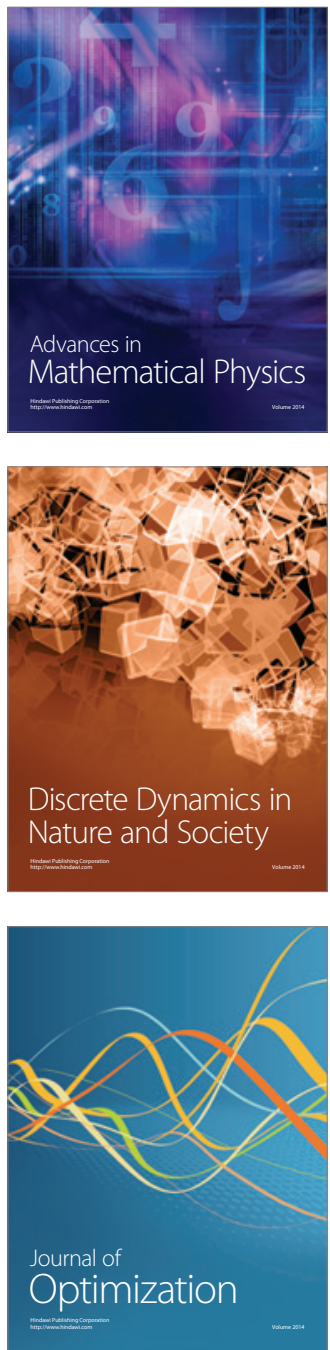